\title{
A NEW OPERATIONAL MODEL FOR SATELLITE-DERIVED IRRADIANCES: DESCRIPTION AND VALIDATION
}

\author{
RICHARD PEREZ ${ }^{\dagger, *}$, PIERRE INEICHEN**, KATHY MOORE*, MAREK KMIECIK*, \\ CYRIL CHAIN $^{* * *}$, RAY GEORGE ${ }^{* * * *}$ and FRANK VIGNOLA ${ }^{* * * * *}$ \\ *ASRC-The University at Albany, Albany, NY, USA \\ **CUEPE-University of Geneva, Geneva, Switzerland \\ ***ENTPE, Vaulx-en-Velin, France \\ $* * * *$ NREL, Golden, CO, USA \\ $* * * * *$ University of Oregon, Eugene, OR, USA
}

Accepted 13 November 2002

\begin{abstract}
We present a new simple model capable of exploiting geostationary satellite visible images for the production of site/time-specific global and direct irradiances The new model features new clear sky global and direct irradiance functions, a new cloud-index-to-irradiance index function, and a new global-to-directirradiance conversion model. The model can also exploit operationally available snow cover resource data, while deriving local ground specular reflectance characteristics from the stream of incoming satellite data. Validation against 10 US locations representing a wide range of climatic environments indicates that model performance is systematically improved, compared to current visible-channel-based modeling practice.

(C) 2003 Elsevier Science Ltd. All rights reserved.
\end{abstract}

\section{INTRODUCTION}

Geostationary satellites monitor the state of the atmosphere and the earth's cloud cover on a space-and-time continuous basis with a ground resolution approaching $1 \mathrm{~km}$ in the visible range. This information can be used to generate time/ site-specific irradiance data and high-resolution maps of solar radiation. Compared to ground measurements, satellite-derived hourly irradiances have been shown to be the most accurate option beyond $25 \mathrm{~km}$ from a ground station (Zelenka et al., 1999). Another noted strength of the satellite resides in its ability to accurately delineate relative differences between neighboring locations, even though absolute accuracy for any given point may not be perfect; hence satellites have proven to be a reliable source of solar microclimate characterization.

Models capable of exploiting satellite observation to generate irradiances range from physically rigorous to purely empirical. At the one end, physical models attempt to explain the observed earth's radiances (the brightness seen by the satellite) by solving radiation transfer equationsthis requires absolute satellite calibration knowl-

"Author to whom correspondence should be addressed; e-mail: perez@asrc.cestm.albany.edu edge and precise information on the composition of the atmosphere. At the other end, empirical models may consist of a simple regression between satellite count and a corresponding measurement at the earth's surface. Hybrid models of the type considered here use a simple physical modeling approach and some degree of fitting to observations. Extensive reviews and discussion on this subject may be found, e.g., in Schmetz (1989), Noia et al. (1993), Pinker et al. (1995) and Perez et al. (2001).

Simple satellite models derive a cloud index (CI) from the satellite visible channel and use this index to modulate a clear sky global irradiance model that may be adjusted for ground elevation and atmospheric turbidity. In this paper we present an evolution of such a simple satellite model (Zelenka et al., 1999)—itself based on earlier work by Schmetz (1989) and Cano et al. (1986) — with the objective of addressing observed remaining weaknesses.

\section{OLD MODEL}

\subsection{Global irradiance (GHI)}

This model is an evolution of the original Cano et al. (1986) model, based upon the observation that shortwave (i.e. solar) atmospheric transmissivity is linearly related to the earth's planetary albedo (Schmetz, 1989) sensed by the satellite as 
earth's radiance and reported as an image-pixel count.

The model includes two distinct parts:

1. pixel-to-cloud index (CI) conversion;

2. CI to global irradiance conversion.

2.1.1. Pixel-to-cloud index conversion. Image pixels are received as 'raw' pixels which are proportional to the earth's radiance sensed by the satellite. A raw pixel is first normalized by the cosine of the solar zenith angle to account for first order solar geometry effect. This normalized pixel is then gauged against the satellite's pixel dynamic range (Fig. 1) at that location to extract a cloud index. The dynamic range represents the range of values a normalized pixel can assume at a given location from its lowest (darkest pixel, i.e. clearest conditions) to its highest value (brightest pixel, i.e. cloudiest conditions). The dynamic range at a given location is maintained by the flux of incoming normalized pixels at that location. While the upper bound of the range remains constant (except for a time-line modulation to account for satellite's calibration drift), the lower bound evolves over time as a function of the local ground albedo variations (chiefly snow, moisture, and vegetation effects). Incoming pixels within a sliding time window are used to determine this lowest bound. The old model uses an 18-day window in summer and a shorter 5-day window in winter in an attempt to capture fast evolving snow cover variations. The lower bound is determined as the average of the 10 lowest pixels in the sliding time window. Before being considered for dynamic range maintenance, an incoming pixel is subjected to a secondary normalization to account for a secondary atmospheric air mass effect and for the hot spot effect (Zelenka et al., 1999). The latter is a function of the sun-satellite angle and incorporates both atmospheric back-scatter brightness intensification and the fact that ground surface becomes brighter as the sun-satellite angle diminishes due to the reduction of ground shadows seen by the satellite (e.g. Pinty and Verstraete, 1991). This secondary normalization is then applied in reverse to the lower bound of the dynamic range before it can be compared to an incoming normalized pixel for the determination of the cloud index as

$\mathrm{CI}=\left(\right.$ norpix $\left.-\mathrm{low}^{*}\right) /\left(\mathrm{up}-\mathrm{low}^{*}\right)$

where norpix is the cosine-normalized pixel given by:

norpix $=$ pix am soldist,

where pix $^{1}$ is the 'raw' untreated image pixel, where am is the optical airmass and where soldist is the square of the normalized earth-sun distance.

\footnotetext{
${ }^{1}$ Depending on its source the value of pix may need additional corrections to reflect the original satellite measurement count. In the US, for instance, data available through Unidata (2002), a quadratic correction is applied to visible images for better image viewing. This correction should be removed as part of the pixel normalization process.
}

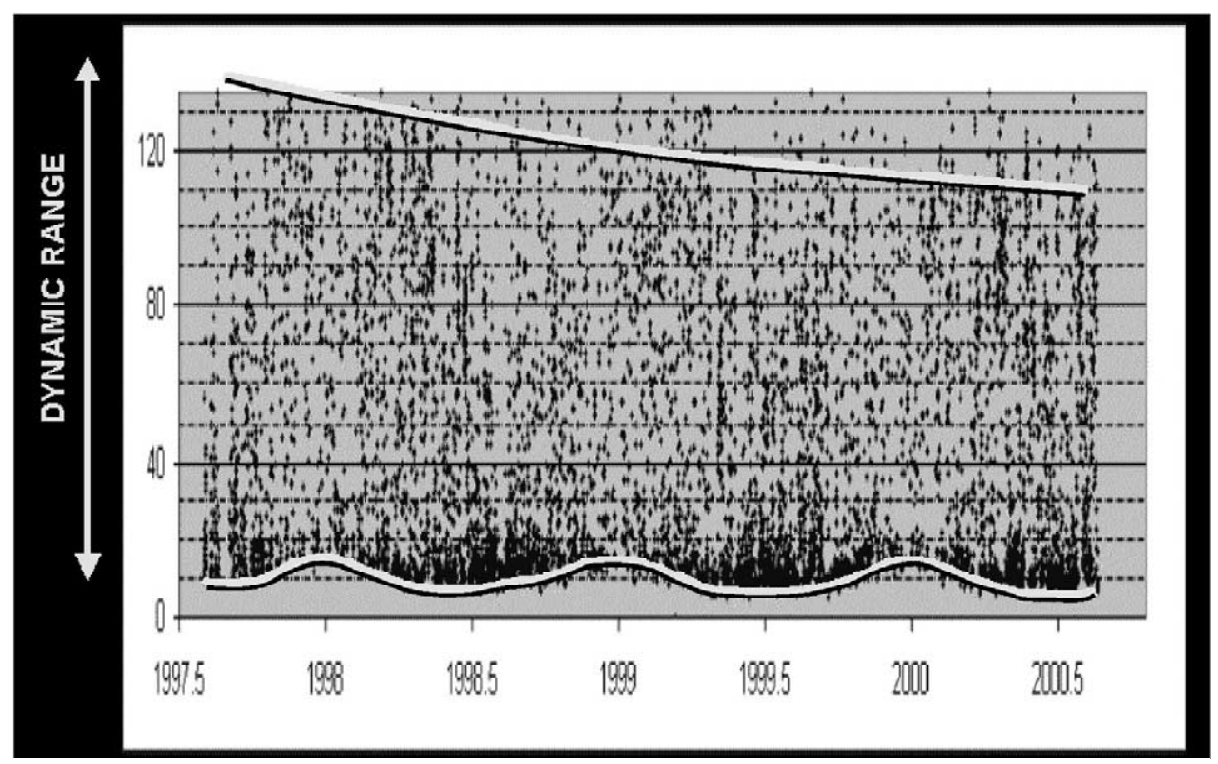

Fig. 1. Satellite dynamic range-GOES-8 southeastern US, 1997-2000. Note the lower bound seasonal variation and the upper bound decrease from satellite calibration decay. 
The parameter up is the dynamic range's upper bound and low* is the lower bound after reverse secondary normalization.

\subsubsection{Cloud-index-to-GHI conversion. GHI is} determined by:

$\mathrm{GHI}=(0.02+0.98(1-\mathrm{CI})) \mathrm{Ghc}$

where Ghc is the clear sky global irradiance per Kasten (Kasten, 1984). Ghc is adjustable for broadband turbidity as quantified by the Linke turbidity coefficient (Kasten, 1980), and ground elevation

$$
\begin{aligned}
\mathrm{Ghc}= & 0.84 I_{\mathrm{o}} \cos Z \exp \{-0.027 \mathrm{am}[\mathrm{fh} 1 \\
& +(\mathrm{TL}-1) \mathrm{fh} 2]\}
\end{aligned}
$$

with

fh1 $=\exp (-$ alt $/ 8000)$

fh2 $=\exp (-$ alt $/ 1250)$

where $I_{\mathrm{o}}$ is the extraterrestrial normal incident irradiance, $Z$ is the solar zenith angle, am is the elevation-corrected air mass, TL is the Linke turbidity coefficient and alt is the ground elevation in metres.

\subsection{Direct irradiance (DNI)}

DNI is modeled from the satellite-derived global using the model DIRINT originally developed and validated for ASHRAE (Perez et al., 1992). This model is an evolution of NREL's DISC model, using a 'stability index' derived from the consecutive records of GHI input.

\subsection{Observed shortcomings of the old model}

- Model bias: although overall bias for GHI has generally been found to be acceptable, there remain important seasonal and regional disparities.

- Snow cover: the short winter-time sliding window to detect rapid albedo changes caused by snow cover in northern locations leads to diminished model performance, sometimes resulting in large winter biases.

- DNI: DNI is extracted from global using a secondary model that had not been developed to fully account for regional turbidity and ground elevation.

- Climate: although the model works relatively well overall in 'generic' temperate climates, limitations have been observed in more extreme climates, particularly in very clear arid locations found in the southwestern US, where the models tend to underestimate irradiances (in particular DNI).

\section{NEW MODEL}

\subsection{Pixel to cloud index}

The new model features two major evolutions: (1) the utilization of external information for snow cover and (2) an accounting of sun-satellite angle effects individualized for each pixel. A seasonal trend adjustment of the dynamic range's lower bound and a minor modification of the secondary airmass effect normalization are also introduced.

3.1.1. Snow cover. For the USA and parts of Canada, the NOHRSC (2002) maintains a daily report of ground snow cover that is accessible via the Internet. The data are made available on a grid of resolution comparable to our satellite archive. The data may be of three types. (a) No snow cover, (b) snow cover and (c) too cloudy to tell. The satellite model uses this information by resetting the value of the dynamic range's lower bound if a pixel's location switches from no-snow cover to snow cover. This implies dropping the current lower bound and replacing it by the value of new incoming lowest pixels. As these pixels get lower as snow ages and melts, the lower bound naturally regains its snow-free value. This process is illustrated in Fig. 2.

3.1.2. Dynamic range lower bound. Using the external snow cover information frees the model to use a longer time window for the dynamic range in winter. The current model uses a yearround 60-day window which allows a robust determination of the lower bound with many data points. The actual minimum, low, is the average of the 40 lowest normalized pixels over this time window. As before, the pixels considered for dynamic range maintenance incoming pixels are subjected to a secondary normalization to account for high air mass effects, leading to a fully normalized pixel npix,

npix $=\operatorname{norpix} /\left(2.283 h^{-0.26} \mathrm{e}^{0.004 h}\right)$

where $h$ is the solar elevation in degrees. Further, $h$ is set at a minimum value of $1.5^{\circ}$ and a maximum value of $65^{\circ}$ for solar elevation outside this interval.

This secondary normalization is then applied in reverse, to the dynamic range's lower bound before comparison with incoming cosine-normalized pixels for the determination of the cloud index.

The switch to a longer year-round window was facilitated by introducing a small trend correction 


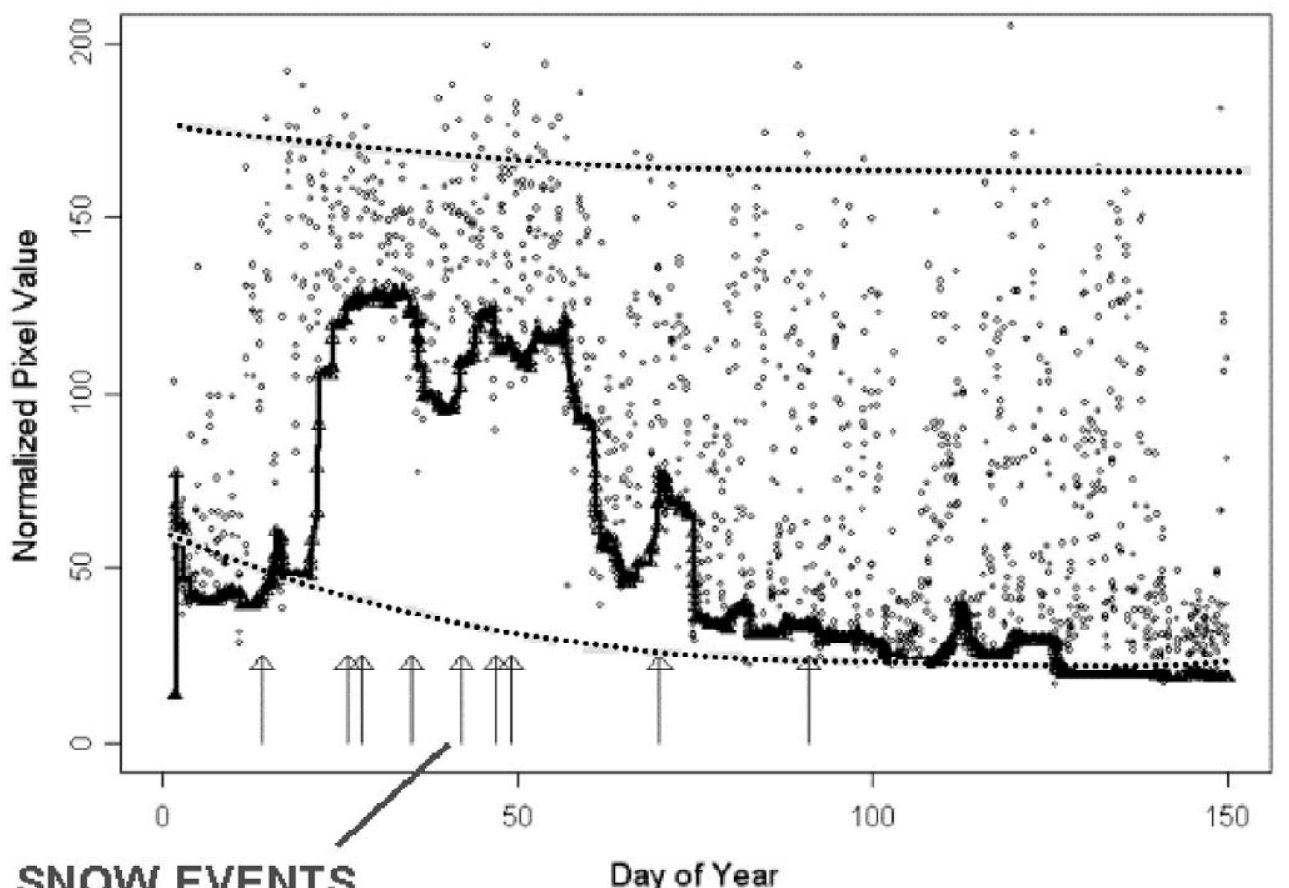

Fig. 2. Impact of snow on the dynamic range's lower bound in Burns, OR, January-May 1999. In the absence of external information on snow cover, the dotted line would be used as the dynamic range's lower bound by the model, resulting in marked underestimation. The snow events force the lower bound to re-initialize based upon the value of incoming pixels resulting in the black lower bound trace.

$\zeta$, based upon the observed mean seasonal variation of the lower bound (e.g. see the seasonal trend in Figs. 1 and 3).

Trend corrected low $=\zeta$ low, with $\zeta=(3+0.5$ $\cos (\operatorname{doy} \pi / 365) /(3.0+0.5 \cos (($ doy $-\operatorname{win} / 2) \pi /$ 365)) where doy is the day of year, and win is the time window length in days.

3.1.3. Sun-satellite angle effects. In the old model we had attempted to account for this effect by using a generic normalization function applicable to all pixels. It soon became apparent that there were strong differences from pixel to pixel, associated with ground cover and soil type. The largest cause of these differences had been overlooked: specular reflectivity of the ground surface. This effect is particularly significant in dry western regions of North America and can vary substantially over short distances. Fig. 3 compares the minimum dynamic range traces for Albuquerque, NM, for a morning and a mid-afternoon hour. Using a single lower-bound trace for all points, as in the old model, led to strong midafternoon underestimations because naturally brighter afternoon pixels were misinterpreted as having a higher cloud index. This shortcoming was resolved by deriving a unique, different function for each pixel relating each hour's rela- tive minimum to the day's lower bound. Operationally, these individualized pixel functions consist of month-by-hours look-up tables derived from several years of archived satellite data. For a given hour/month this factor, Matfac, is equal to:

Matfac $=\operatorname{low}_{1} /$ low,

where low $_{1}$ is the dynamic range's lower bound for the considered hour and month, and low is the lower bound for all hours for the considered month. The factor Matfac is used as a multiplicator to the dynamic range's lower bound before comparison with incoming pixels for the determination of the cloud index.

\subsection{GHI generation}

As in the old model, cloud indices are used to modulate a clear sky global irradiance model that may be adjusted for both broadband turbidity and ground elevation. However, several modifications have been introduced.

3.2.1. Broadband turbidity. Ineichen and Perez (2002) recently proposed a revised formulation of the Linke turbidity coefficient to remove its dependence on solar geometry. This new formulation was used to generate a seasonal grid of TL for the North American continent, based upon 


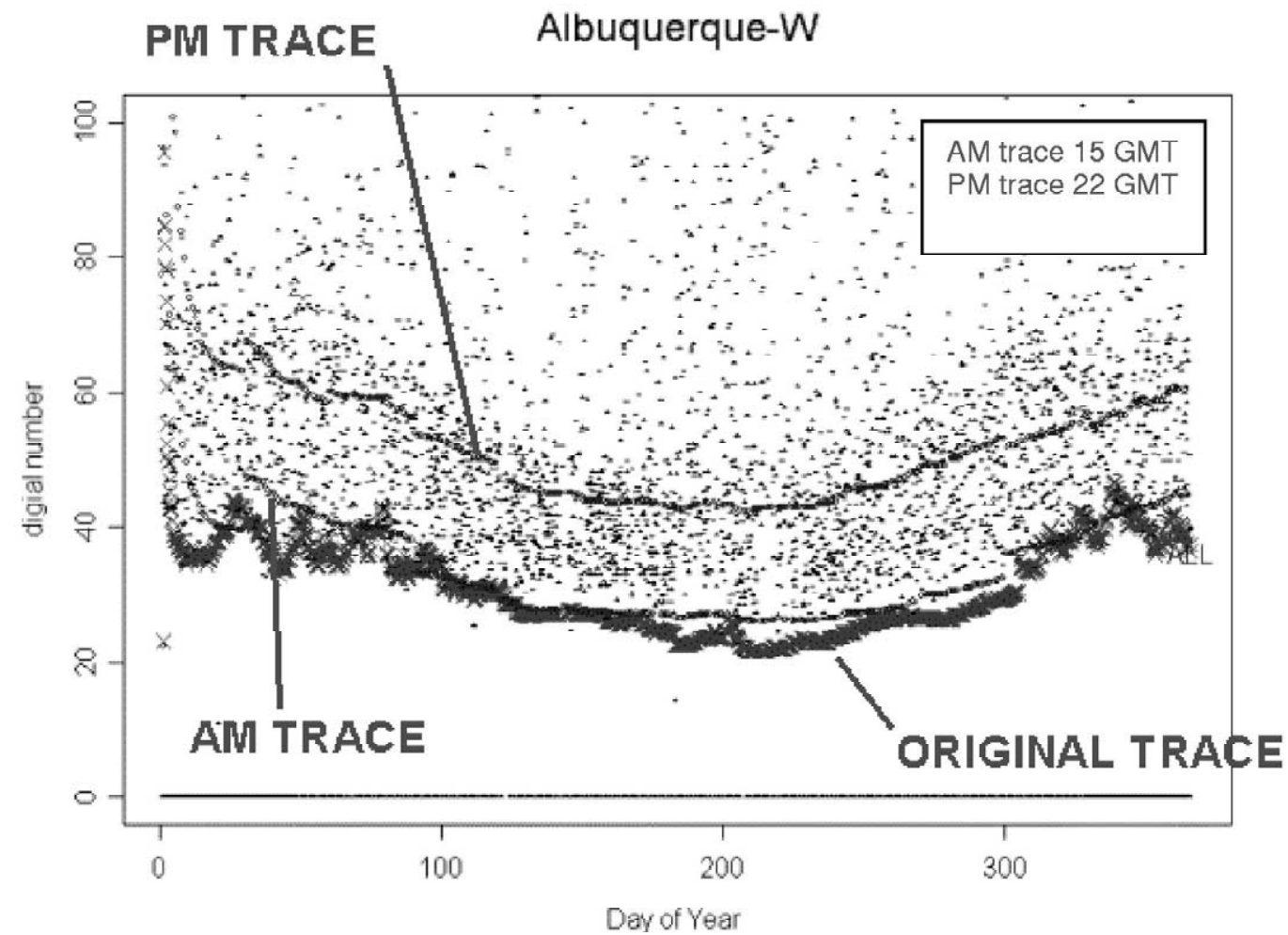

Fig. 3. Impact of ground specular reflectivity on lower bound in Albuquerque-GOES-West data. The PM trace is well above the AM trace because the arid ground appears considerably brighter when the sun satellite reaches a minimum in the afternoon. The original model would infer the lower bound from all the points (original trace), leading to irradiance underestimation in the afternoon as clear pixels would be interpreted as having significant cloud indices.

gridded climatological aerosol, ozone and water vapor data that had previously been assembled for the preparation of the NSRDB (1995). The new formulation could also be used, as appropriate, to generate TL 'on the fly' from regional ground monitoring stations (e.g. from DNI measurements).

\subsubsection{Clear-sky global irradiance. The Kasten} clear sky model was modified (Ineichen and Perez, 2002) to exploit the new turbidity formulation and to improve its fit of observed data for very clear/high elevation locations found in the western part of the continent, while conserving its representativeness of standard temperate environments

Ghcnew $=\operatorname{cg} 1 I_{\mathrm{o}} \cos z \exp (-\operatorname{cg} 2 \mathrm{am}(\mathrm{fh} 1$

$$
+\mathrm{fh} 2(\mathrm{TL}-1))) \exp \left(0.01 \mathrm{am}^{1.8}\right)
$$

with

cg1 $=(0.0000509$ alt +0.868$)$

cg2 $=0.0000392$ alt +0.0387 .

3.2.3. Cloud-index function. The linear CI-toGHI function was dropped in favor of a form representative of observed data. The present formulation, plotted in Fig. 4, is a fit to five environmentally distinct, very high quality ground truth stations (Albany, NY, Burlington, KS, Eugene, Gladstone and Hermiston, OR).

$\mathrm{GHI}=\mathrm{Ktm}$ Ghcnew $(0.0001 \mathrm{Ktm}$ Ghcnew +0.9$)$

with

$$
\begin{aligned}
\mathrm{Ktm}= & 2.36 \mathrm{CI}^{5}-6.2 \mathrm{CI}^{4}+6.22 \mathrm{CI}^{3} \\
& -2.63 \mathrm{CI}^{2}-0.58 \mathrm{CI}+1 .
\end{aligned}
$$

Differences with the simple original linear function are not very large. Nevertheless the experimental data do reveal a definite trend, with maximum departures at the upper and lower ends of the curve. At the lower end-high CI-the new function produces noticeable performance improvement for low irradiances (e.g. see Fig. 6, global irradiance). At the upper end-low CI-the curvature reflects the fact that some uncertainty exists in the exact location of the clear pixel's edge (e.g. see the lower bound points in Fig. 1).

\subsection{DNI generation}

DNI is obtained, as GHI, via modulation of clear-sky direct irradiance. The clear sky ir- 


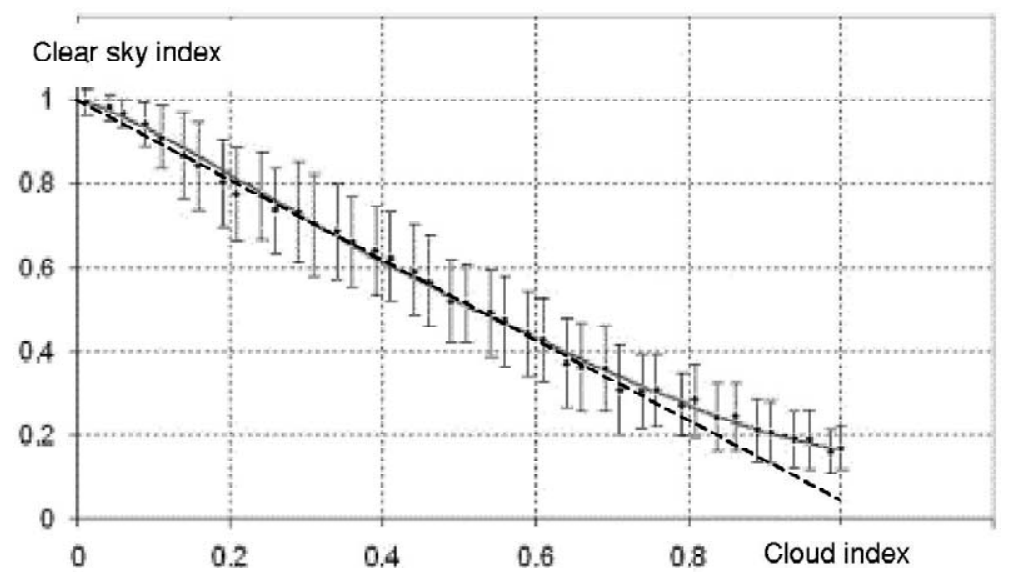

Fig. 4. Illustration of the new CI-to-GHI function fitted to all available data. The dotted line illustrates the linear function of the original model. The largest departure is observed for high cloud indices, showing that the original model tended to underestimate irradiance for high pixel brightness. A small difference is also noticeable for very low indices, showing that the original model tended to underestimate irradiance-this small underestimation tendency was compounded when extracting direct irradiance from global.

radiance model was recently introduced by the authors as part of the development of the new TL formulation (Ineichen and Perez, 2002):

$$
\begin{aligned}
\mathrm{Bc}= & \operatorname{Min}\left\{0.83 I_{\mathrm{o}} \exp (-0.09 \mathrm{am}[\mathrm{TL}-1])(0.8\right. \\
& +0.196 / \mathrm{fh} 1),
\end{aligned}
$$$$
\left.\left(\text { Gcnew }-D_{\mathrm{c}}\right) / \cos Z\right\}
$$

where $D_{\mathrm{c}}$ is the minimum clear sky diffuse irradiance given by:

$$
\begin{aligned}
D_{\mathrm{c}}= & \text { Gcnew }\{0.1[1-2 \exp (-\mathrm{TL})]\}\{1 /[0.1 \\
& +0.882 / \mathrm{fh} 1]\} .
\end{aligned}
$$

Unlike GHI, the direct modulating factor is not derived from CI as we had originally expected (Ineichen and Perez, 1999) but from global, using the global-to-DNI model, DIRINT (Perez et al., 1992), in a relative mode. In this relative mode, DIRINT is used twice, once to calculate an intermediate direct irradiance, $\mathrm{DNI}_{1}$, from GHI and, a second time to calculate an intermediate clear sky direct irradiance, $\mathrm{DNI}_{2}$, from Ghcnew. DNI is obtained from:

$\mathrm{DNI}=\mathrm{Bc}\left[\mathrm{DNI}_{1} / \mathrm{DNI}_{2}\right]$.

This approach retains the main strength of DIRINT: the use of a stability index derived from the GHI data stream (Perez et al., 1990) while it mitigates its main weakness: the lack of calibration of this model with respect to a standard clear sky profile.

\subsection{Operational model}

While the modeling process has been thoroughly described above, the operation of the model on a geographic scale, either for the preparation of maps or site/time-specific time series requires some degree of logistics and information processing. This logistical approach is summarized in Fig. 5. It includes several layers of gridded information. The grid size of our current archive is 0.1 degrees latitude-longitude. Ultimate achievable resolution of visible channel GOES image could approach 0.01 degree. The information gridded layers include:

- raw satellite pixels (visible channel) obtained via direct processing of primary GOES east and GOES west satellite images. We archive gridded raw pixel frames on an hourly basis;

- terrain elevation;

- climatological Linke turbidity-12 monthly layers, derived from previously gridded aerosol optical depth data (NSRDB, 1995);

- snow cover-daily gridded frames from NOHRSC (2002);

- specular correction factor-216 layers (12 months by $18 \mathrm{~h}$ ) derived from the hourly processing of 5 years worth of raw pixel data (see sun-satellite angle effects, above).

\section{VALIDATION}

\subsection{Experimental ground truth data}

A total of 10 stations, listed in Table 1 are used to evaluate model performance. As mentioned 


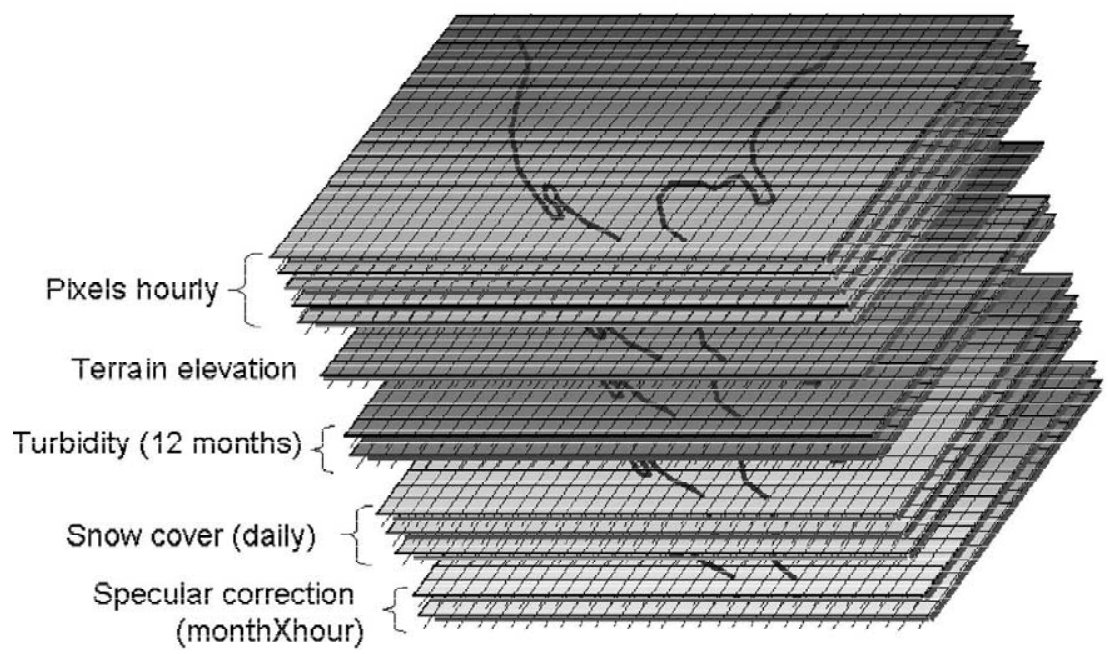

Fig. 5. This figure illustrates all the geographically gridded data sets that are used operationally in the North-American model, including hourly or $1 / 2$ hourly image pixels, terrain elevation, monthly Linke turbidity, daily snow-cover updates and the ground specular reflectivity correction factors (monthly-hourly averages).

above, data from five of these stations (*) were used to fit the CI-to-global transfer function. Most of the stations follow rigorous calibration and quality control protocols, particularly those directly or indirectly affiliated with the ARM (2002) or BSRN (2002) programs and with the Pacific Northwest network.

Albany, ARM-Burlington and FSEC are used to validate irradiances derived from GOES-East (GOES-8) data. Burns, Eugene, Gladstone, Hermiston, Klamath Falls and Kramer Junction are used for GOES-West (GOES-10). Albuquerque is used to validate irradiances derived from both satellites.

\subsection{Results}

The first set of validation metrics-overall observed root mean square errors (RMSE) and mean bias errors (MBE) - is presented in Table 2 . We compare the old model against two versions of the new model: (1) the operational model as described in this paper, and (2) the same with monthly turbidity derived locally from the ground truth DNI measurements. The statistics are based on $96 \%$ of the points, rejecting $2 \%$ of the most extreme positive and negative differences-most of the highest differences have little to do with model intrinsic accuracy but are mostly reflective of the impact of cloud patterns on the comparison between an instantaneous measurement extended in space-the satellite pixel-and a pinpoint ground measurement extended in time-hourly integration (Zelenka et al., 1999).

The observed performance improvement is systematic for all sites using the RMSE benchmark. This is remarkable since the room for gain is not as large as the magnitude of the initial error would suggest. Because of the above-mentioned pixel-ground station discrepancy and small remaining satellite navigation uncertainties the initial accuracy is already close to achievable effective accuracy (Zelenka et al., 1999). As it is, the new model approaches this limit for GHI at several of the sites. Inspection of the MBE

Table 1. Ground truth stations

\begin{tabular}{lllcl}
\hline Site & Climate & Latitude $\left(^{\circ}\right)$ & Longitude $\left(^{\circ}\right)$ & Notes \\
\hline Albany, NY (1999) & Humid continental & 42.7 & -73.8 & BSRN (2002) \\
Burns, OR (1999-2000) & Semi-arid, high elevation & 43.6 & -119.1 & Pacific Northwest network \\
Albuquerque, NM (1999) & Arid, high elevation & 35.1 & -106.6 & Sandia Natl. Labs, ARM protocol \\
ARM-Burlington, KS (1999) & Dry continental & 38.1 & -95.7 & ARM-SGP extended facility \\
Eugene, OR (1999) & Temperate & 44.0 & -123.1 & Pacific Northwest network \\
FSEC-Cocoa, FL (1999) & Subtropical & 28.3 & -80.7 & Florida Solar Energy center \\
Gladstone, OR, (part-1999) & Temperate, humid & 45.3 & -122.6 & Pacific Northwest network \\
Hermiston, OR (1999-2000) & Temperate, dry & 45.8 & -119.3 & Pacific Northwest network \\
Klamath Falls, OR (pt-1999) & Temperate, dry & 42.2 & -121.8 & Pacific Northwest network \\
Kramer Junction, CA (1999) & Arid & 35.0 & -117.5 & SEGS power plant monitoring \\
\hline
\end{tabular}


Table 2. Model RMSE and MBE for global and direct irradiance

\begin{tabular}{|c|c|c|c|c|c|c|c|}
\hline \multirow{2}{*}{$\begin{array}{l}\text { Day-time avg. } \\
\left(\mathrm{W} \mathrm{m}^{-2}\right)\end{array}$} & & \multicolumn{3}{|c|}{ RMSE } & \multicolumn{3}{|l|}{ MBE } \\
\hline & & Old & New & New* & Old & New & New* \\
\hline \multicolumn{8}{|l|}{ Global } \\
\hline Albany & 326 & 72 & 68 & 69 & -7 & -11 & -1 \\
\hline Burns-99 & 391 & 89 & 79 & 79 & -20 & -21 & -20 \\
\hline Burns-00 & 383 & 69 & 62 & 62 & 2 & 1 & 2 \\
\hline Albuq. (Goes-E) & 485 & 76 & 69 & 69 & -31 & -3 & -5 \\
\hline Albuq. (Goes-W) & 486 & 90 & 80 & 80 & -22 & 1 & -1 \\
\hline ARM-Burlington & 355 & 57 & 51 & 51 & 6 & -1 & 2 \\
\hline Eugene & 311 & 64 & 53 & 53 & 3 & 7 & 4 \\
\hline FSEC-Cocoa & 421 & 121 & 115 & 118 & 34 & 31 & 52 \\
\hline Gladstone & 290 & 64 & 60 & 60 & 18 & 17 & 24 \\
\hline Hermiston-99 & 358 & 52 & 45 & 45 & -6 & 2 & -2 \\
\hline Hermiston-00 & 357 & 47 & 44 & 44 & 7 & 15 & 11 \\
\hline Klamath-Falls & 357 & 58 & 47 & 50 & 18 & 7 & 16 \\
\hline Kramer Junction & 487 & 69 & 48 & 49 & -19 & -11 & 8 \\
\hline All sites & 385 & 61 & 54 & 54 & -4 & 0 & 3 \\
\hline \multicolumn{8}{|l|}{ Direct } \\
\hline Albany & 345 & 165 & 154 & 155 & 5 & -40 & -3 \\
\hline Burns-99 & 483 & 204 & 190 & 188 & -33 & -41 & -35 \\
\hline Burns-00 & 477 & 198 & 172 & 171 & -1 & -5 & 0 \\
\hline Albuq. (Goes-E) & 629 & 179 & 169 & 165 & -84 & -4 & -19 \\
\hline Albuq. (Goes-W) & 629 & 205 & 187 & 185 & -64 & 26 & 8 \\
\hline ARM-Burlington & 397 & 131 & 121 & 117 & 5 & -42 & -28 \\
\hline Eugene & 305 & 158 & 116 & 112 & 18 & 23 & 7 \\
\hline FSEC-Cocoa & 339 & 209 & 193 & 207 & 62 & 36 & 100 \\
\hline Gladstone & 276 & 151 & 118 & 122 & 38 & 11 & 48 \\
\hline Hermiston-99 & 494 & 151 & 133 & 129 & -19 & 21 & 2 \\
\hline Hermiston-00 & 434 & 163 & 138 & 135 & -17 & 25 & 5 \\
\hline Klamath-Falls & 493 & 199 & 163 & 174 & 33 & -17 & 31 \\
\hline Kramer Junction & 672 & 231 & 156 & 161 & -104 & -83 & 27 \\
\hline All sites & & 161 & 137 & 137 & -19 & -11 & 4 \\
\hline
\end{tabular}

Note, the new column corresponds to climatological turbidity, while new* corresponds to local measurements-derived turbidity.

benchmark also reveals overall improvementnote in particular that the strongest underestimations (Albuquerque, Kramer) and overestimations (Gladstone) have been reduced. The model using local measurement-based turbidity shows only slight additional improvement. The case of Florida stands out, with a much stronger initial RMSE and MBE and small accuracy improvement. The causes for this will have to be investigated further. At present the two major suspected causes are: (1) the humid subtropical climate with frequent broken cloud patterns, and more likely, (2) the fact that Cocoa is situated at the edge of a body of water, with a satellite pixel straddling two very different minimum brightness environments.

Model performance improvement may be qualitatively visualized in Figs. 6 and 7 that compare the old and the new (with generic TL) performance in Albuquerque, NM for GOES west. Note that Albuquerque provides a fully independent model test bed as this site was not used to fit the CI-to-GHI index function. The reduction of scatter and high-end bias is particularly striking for DNI. Much of the improvement at that site stems from the utilization of the pixel-specific look-up table describing the local sun-satellite angle effect. In the old model, enhanced brightness of the ground peaking in early afternoon is mistaken as increased cloud index resulting in a severe DNI underestimation. Much of this shortcoming is corrected with the new model.

Overall bias may appear reasonable only to hide seasonal effects that may cancel-out. So, another gauge of model performance improvement is to observe the variations of seasonal biases. Fig. 8 compares the seasonal DNI bias traces for all sites. The new model traces are noticeably more compact showing more site-tosite as well as season-to-season consistency.

\section{CONCLUSION}

We have presented a new simple model capable of exploiting geostationary satellite visible images for the production of site/time-specific global and direct irradiances. The model exhibits systematic performance improvement for all tested locations representing a wide range of climatic environments. As it is, the level of observed RMSE at some of the test stations approaches the effective 

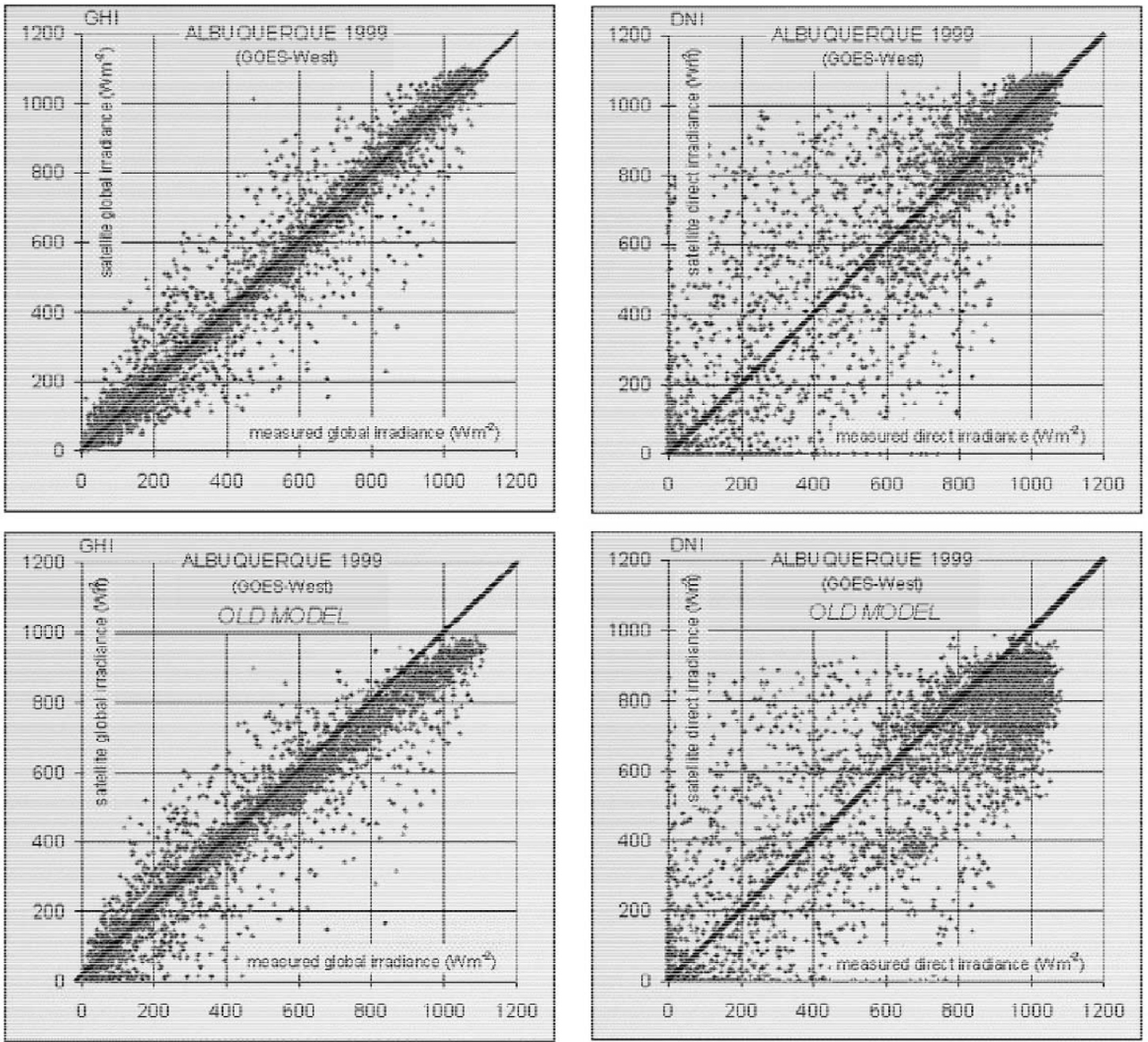

Fig. 6. Modeled vs. measured hourly global irradiance for the old and new model in Albuquerque using GOES-West as model input. Model performance improvement at the high end results from taking specular ground reflectivity into account. Improvement at the low end results from a better CI fit (see Fig. 3).

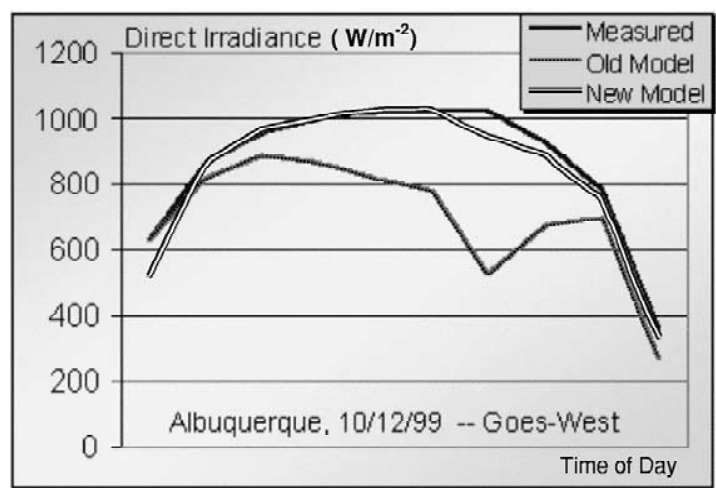

Fig. 7. Comparing measured and modeled typical clear-sky DNI daily profiles in Albuquerque, NM. The original model's deficit peaks in mid afternoon when the local sun-satellite angle reaches its minimum value. accuracy limit previously discussed by the authors and co-workers (Zelenka et al., 1999). The new model is particularly efficient at correcting possible distortions resulting from certain types of ground surfaces.

Future work will focus on (1) addressing remaining ground specularity effects that may leave a trace in the production of microclimatic solar resource maps; (2) investigating other climates, particularly subtropical and tropical, which have only been marginally covered here; (3) investigating whether additional satellite channels (in particular IR) may lead any noticeable cloud index detection and model performance improvement; (4) comparing this model with other models (e.g. Broesamle et al., 2001) using common ground truth stations as will been done as part of the SWERA program (SWERA, 2002). 


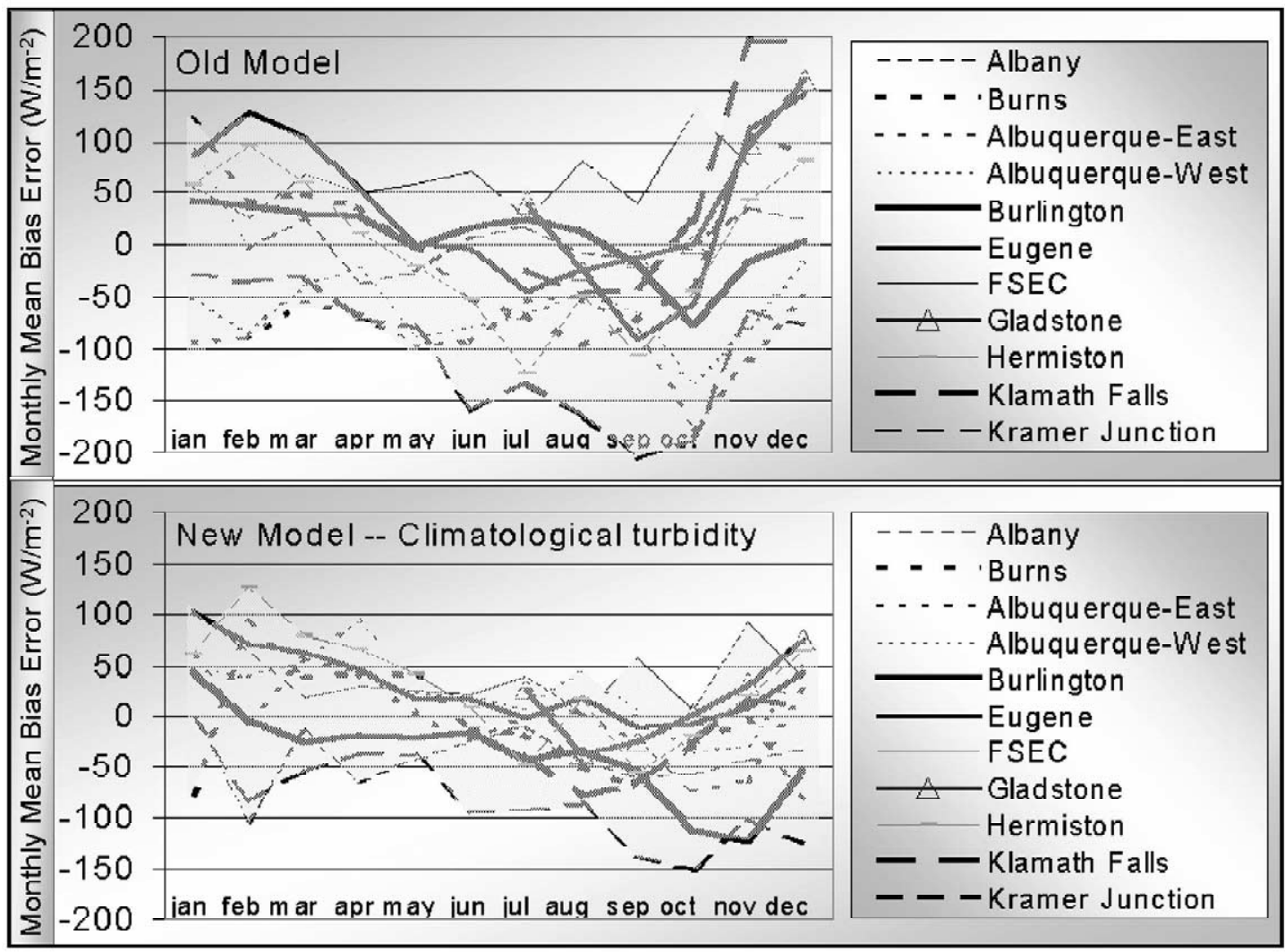

Fig. 8. Comparing old and new model mean bias error monthly trends for direct irradiance for all stations.

Acknowledgements-This work combines the research and findings of two programs: NREL contract NAA-13044102 and University of Oregon's Solar Resource GIS Data Base for the Pacific Northwest (no. 280111A) and constitutes initial validation effort for UNEP's SWERA program. Many thanks to Antoine Zelenka (Meteo-Suisse) and to Qi Long Min (ASRC) for their helpful discussions along the way, to Bill Boyson (Sandia National Labs), Jim Schlemmer and Joe Michalsky (ASRC) for the Ground Truth Data.

\section{REFERENCES}

ARM (2002). Atmospheric Radiation Measurement Program, http://www.arm.gov/.

Broesamle H., Mannstein H., Schillings C. and Trieb F. (2001) Assessment of solar electricity potentials in North Africa based on satellite data and a geographic information system. Solar Energy 70, 1-12.

BSRN (2002). Baseline Surface Radiation Network, http:/ /bsrn.ethz.ch/.

Cano D., Monget J. M., Aubuisson M., Guillard H., Regas N. and Wald L. (1986) A method for the determination of global solar radiation from meteorological satellite data. Solar Energy 37, 31-39.

Ineichen P. and Perez R. (1999) Derivation of cloud index from geostationary satellites and application to the production of solar irradiance and daylight illuminance data Theor. Appl. Climatol. 64, 119-130.

Ineichen P. and Perez R. (2002) A new airmass independent formulation for the Linke turbidity coefficient. Solar Energy 73(3), 151-157.
Kasten F. (1980) A simple parameterization of two pyrheliometric formulae for determining the Linke turbidity factor. Meteorol. Rdsch. 33, 124-127.

Kasten F. (1984) Parametriesirung der globalstahlung durch bedeckungsgrad und trubungsfaktor. Annal. Meteorol. Neue Folge 20, 49-50.

Noia M., Ratto C. and Festa R. (1993) Solar irradiance estimation from geostationary satellite data: I. statistical models, II. physical models. Solar Energy 51, 449-465.

NOHRSC (2002). National Operational Hydrologic Remote Sensing Center, http://www.nohrsc.nws.gov/.

NSRDB (1995). In National Solar Radiation Data Base-Final Technical Report, Vol. 2, NREL/TP-463-5784.

Perez R., Ineichen P., Seals R. and Zelenka A. (1990) Making full use of the clearness index for parameterizing hourly insolation conditions. Solar Energy 45, 111-114.

Perez R., Ineichen P., Maxwell E., Seals R. and Zelenka A. (1992) Dynamic global-to-direct irradiance conversion models. In ASHRAE Transactions-Research Series, pp. 354-369.

Perez R., Aguiar R., Collares-Pereira M., Dumortier D., Estrada-Cajigal V., Gueymard C., Ineichen P., Littlefair P., Lund H., Michalsky J., Olseth J. A., Renné D., Rymes M., Skartveit A., Vignola F. and Zelenka A. (2001) Solar resource assessment-a review. In Solar Energy-The State of the Art, Zelenka A. (Ed.), James \& James, London, Chapter 10, Section 10.4.4.

Pinker R. T., Frouin R. and Li Z. (1995) A review of satellite methods to derive surface shortwave irradiance. Remote Sens. Environ. 51, 108-124.

Pinty B. and Verstraete M. M. (1991) Extracting information on surface properties from bidirectional reflectance measurements, J. Geophys. Res. 96, 2865-2874. 
Schmetz J. (1989) Towards a surface radiation climatology: retrieval of downward irradiances from satellites. Atmos. Res. 23, 287-321.

SWERA (2002). Solar and Wind Resource Assessment, http:/ /www.uneptie.org/energy/act/re/fs/swera.pdf.
Unidata (2002). Unidata Internet Data Distribution System, http://my.unidata.ucar.edu/.

Zelenka A., Perez R., Seals R. and Renné D. (1999) Effective accuracy of satellite-derived irradiance. Theor. Appl. Climatol. 62, 199-207. 